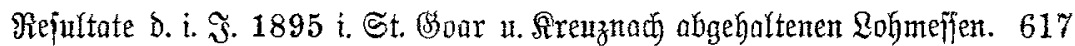

Für Dte einzelnen Sortimente wurben pro Centner nadjtebende Scöchitgebote erzielt:

(s)lanzrinbe

II. " . . . . . . . 4,00"

Reitelrinte

I. น. II. $\mathfrak{R l}$. . . . . 4,50" $" 5,20$ "

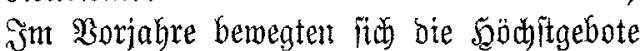

bei Glanzrinde I. Al. . . . . zwithen 4,50-4,80 All

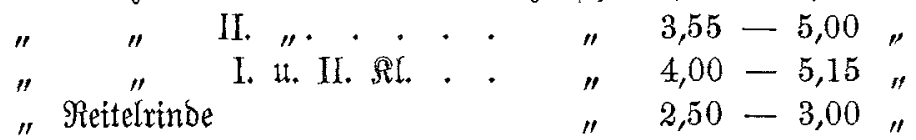

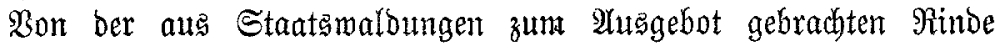
murben ben Fejujitgeboten alf

4459 Str. Blanzrinde I. Rl.

8501

II. " $\mathfrak{u}$.

250 "Reitelrinto

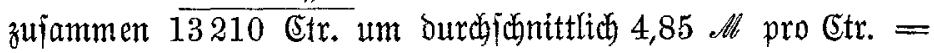

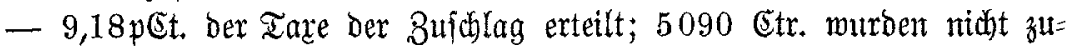

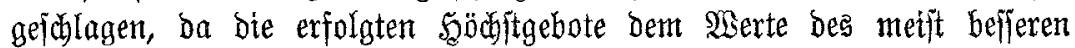
Materials nidat entiprachen.

Die Beteiligung war zablreich und die meiften Grophbändlex waren vertreten. Sämtliche Roje bes Stantsanteiles mit 2Utsnabme zweier ganz unbebentender soje mit 60 (Str. fomnten proviforifd zugejdblagen werben; Doch bemegten fich die \$reije nicht über bem Niveau ber voriäbrigen, ba

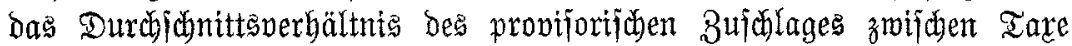

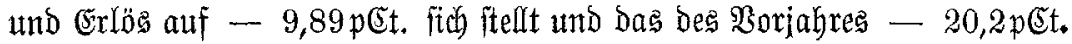
betrug; Das Berbältnis Der betDen Sabre ift trobe Der fibeinbaren Differenz nahezu gleidh, ba bie Taxe bes heurigen sabhres $\mathbf{t m}$ runb 10 pet. ex= nieorigt worben itt.

\title{
Repultate der im Jahre 1895 in St. Goar und Kreuznach abgehaltenen cohmeffen.
}

Die Rngmeffe bes Sreifes St. Boar muroe am 14. März in St. Soar abgebalten. Aluch in biejem Jahre hatten ith ben (Gemeinden bes Fretjes bie Riöniglichen Dberföritereien Eaftellaun, Roblenz und Reupfalz, fowie

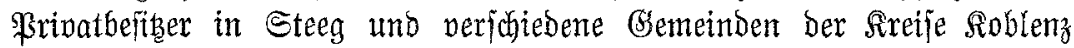
und Mayen angeiditiffen.

§im ganzen wutrben 16096 Ctr. Sobe auggeboten, und zwar 785 aus Röniglichen, 13911 aus Gemeinbe= und 1400 aus Frivatfortten. 


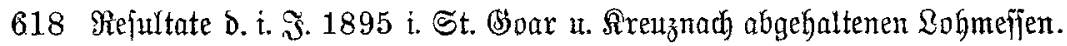

(S)gen bas $\mathfrak{B}$ orjahr erfolgte ein Mebrangebot von 16096 meniger 12455 $=3641$ Ctr.

Bormiegend war bie Dutaltät ber Rinden eine gute. Nut 16 p巨t. (2631 Ctr.) waren über 18 şabre alt. Das alter Der einzelnen હchläge

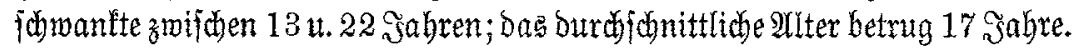

Der Berfauf ging flott von ftatten. Sei eintgermanen annefubaren Rebitgeboten erfolgte Der beftnitive Bujlalag fofort. Rachgebote muroen nidht angenommen. Das Schälen ber Robe bewirfte ber Berfäufer.

Bon bem offeriertent sejammtquantum murben 14251 (str. verfauft. Für 1845 Ctr., Darunter bie won Den Frivatbeftiern in હteeg zum $\mathfrak{B} e r=$ fauf geilellten 1400 Etr., wurbe ber 3 ulfollag nicht erteilt.

Błts 18jäbrige Ritnoen erzieltent einen Durchidjnittspreis von 5 eth

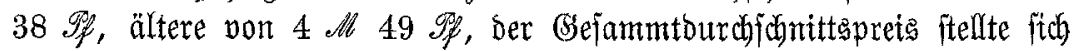

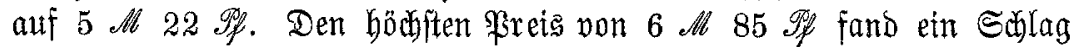

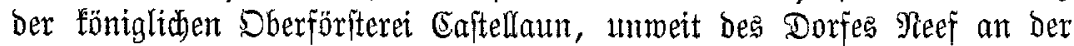
Mojel gelegen, mit 360 Centrern 15 jäbriger Rinden. Der nieorigfte Freis von 3 ath wurde für je einen fleinen Shlag der Stabt Et. Osoar

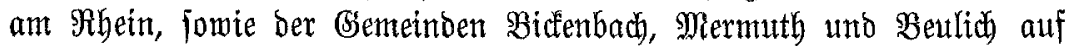

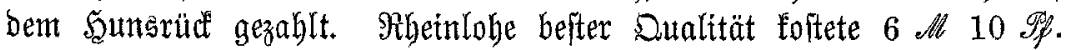

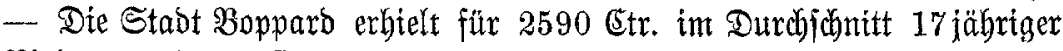
ßinden $5 \mathscr{M} 98 \mathscr{P}$.

Dem getroffenen $\mathfrak{A b f o m m e n ~ g e m a ̈ p ~ f a n o ~ b i e ~ S r e u z n a c h e r ~ \& o h m e f f e ~}$

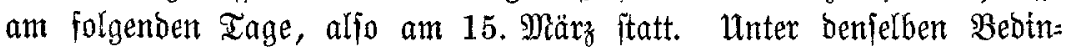
gungen wie in St. Boar wurben 39565 gegen 41820 Štr. int $\mathfrak{B o r j a h r e ~}$ zum Serfauf geftellt, Davon aus Den föniglitben Dberjörftereien (Enten= pfubl und Mieifentheim 3430, aus gröperen Frivatwaldungen 3910, ber Reit aud den Benteindeforiten der Sreife Srelzzach unto Meifenbeim.

Sämtlìbe ßinben waren von guter, meift jogar vorzüglicher Dualität. Sie entftammten 13 bis 18, Durdifontitflich 16 sabre alten \&objolägen.

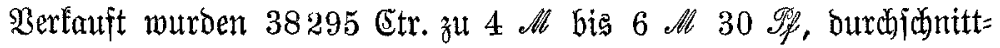

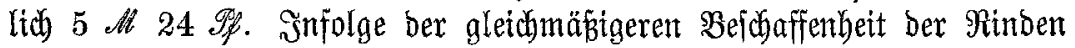
in ben einzelnen SBerfaufalojen waren hier bie \$reisjothwanfungen weit

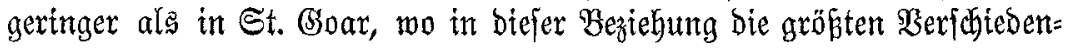
heiten obmalteten. Die beredoneten Bejantmtourdjibnittspreife von $5 \mathscr{M}$

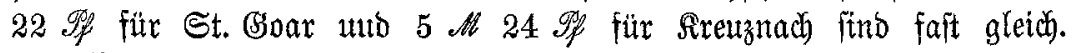
Diejelben betrugen in

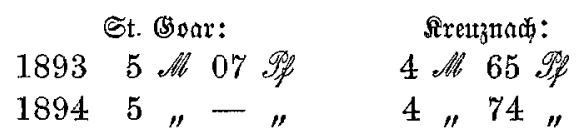

Ěine erfreulidfe $\mathfrak{B}$ endung zum $B$ efferen ift Demmach eingetreten. 


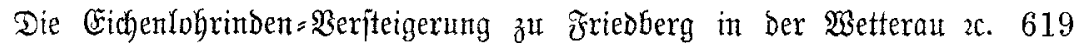

Seitbem bie Mehrheit des beutichen Reidstaçes fich für bie Einführung eines Echubzolles auf Duebradjobolz autsgeiprodhen hat, iit ein allmähliger Alufichung in ber beutiogen Rederinduitrie bemerfbar gemorden. Mit

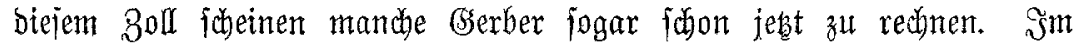
Sntereffe ber Erraltung bes Eichenjojälwaldes und bes Fortbeitebens und weiteren Bebeihens ber Eichenlohegerbereien ift baher ber $\mathfrak{M u m i d} b=$

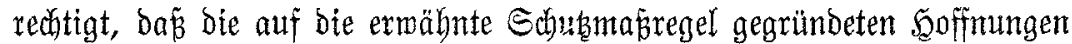
fith voll und ganz erfüllen mögen!

R.

\section{Die Eichenlohrinden = Derfteigerung $\mathfrak{z}^{\mathfrak{u}}$ friedberg in der Wetteran vom Jahre 1895.}

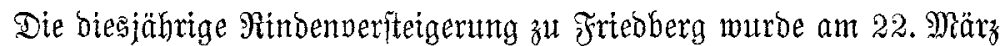
abgebalten. Wie bei allen am htefigen \$slabe bis jebst vorausgegangenen Märtten, muroe auch biesmal burch Sandeln nach ber 2Uluttion bag Şaupt=

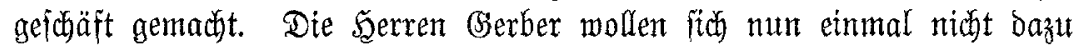
veritehen, ben wirflichen Marlprets bezm. Den Preis, um welchen fie bie

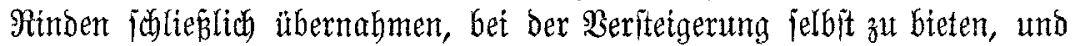

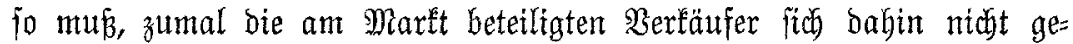

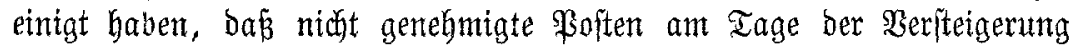
weber mieberbolt auggeboten, nod) aus der Sand verfauft werben bürfen,

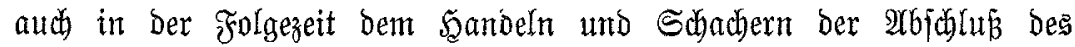
(Bełchäftes biberlaffen werben.

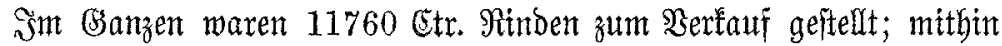
560 Ctr. weniger als im Borjahre. Diefes Mimus bat feinen Grumb

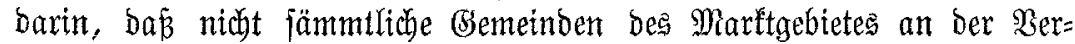
fteigerung teilgenommen haben, weil einzelne berfelben infolge früherer

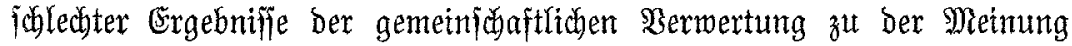
gefommen waren, auf bem $\mathfrak{x e g e}$ Des Einzelverfaufs aus ber Şand beffere

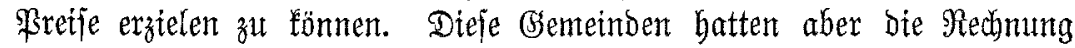
oyne ben Wirt gemadt. Bon Den ausgebotenen Rinden entfallen rund

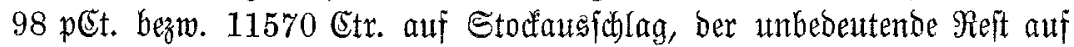

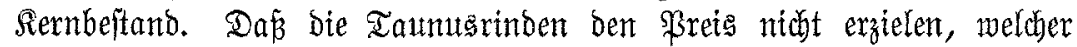

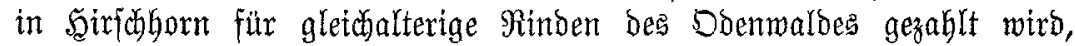

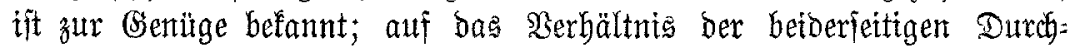

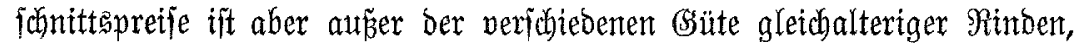
fowie ber ungleichen $2 \mathfrak{u}$ frnabmefäbigleit bes Marftgebtetes, wie Sauffraft

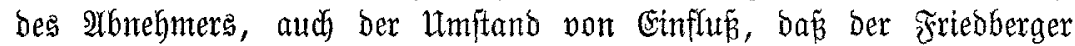

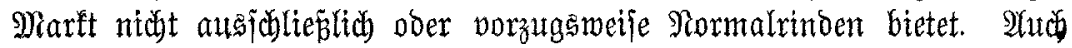

